ISSN: 2599-3496 print

ISSN: 2614-2376 online

\title{
Processing, Characteristics, and Potential Application of Red Palm Oil - a review
}

\author{
Kartika Okta Purnama1, Dwi Setyaningsih ${ }^{1,2^{\star}}$, Erliza Hambali ${ }^{1,2}$, Darmono Taniwiryono ${ }^{3}$ \\ ${ }^{1}$ Agricultural Industrial Engineering, IPB University, Bogor, \\ ${ }^{2}$ Surfactant and Bioenergy Research Center (SBRC), Bogor, \\ ${ }^{3}$ PT Nutri Palma Nabati, Bogor
}

\begin{abstract}
Palm oil contains several nutrients such as carotene and vitamin $E$ (in the form of tocopherols and tocotrienols) which are very beneficial for human health. One of the palm oil products that contain high phytonutrient compounds is red palm oil (RPO). The red color of the oil indicates high carotene content. RPO can be produced from refinery process including degumming, neutralization and deodorization of unrefined palm oil (crude palm oil, CPO). The process of refinery at low temperatures aims to reduce levels of free fatty acids (FFA) which are still relatively high and to keep the levels of phytonutrients such as carotene high. RPO has a distinctive red color because it contains high levels of carotene (200-700 ppm) and low FFA levels $(<1 \%)$. However, carotene levels and FFA levels in RPO are lower when compared to CPO because RPO has undergone heating during the chemical refinery process through the degumming, neutralization, and deodorization process stages. RPO has been used as cooking / stir-frying oil, shortening, margarine, and several other food products. Diversification of RPO-based products is needed so that the nutrients in the oil can be utilized properly. This paper reviews several studies on the processing, characteristics, and potential uses of RPO. The application of RPO in food products, especially margarine, has the potential to be further investigated.
\end{abstract}

Keywords: applications, characteristics, processes, RPO, and VRPO

\section{INTRODUCTION}

Palm oil (Elaeis guineensis) is one of the most important plants in the world because of its high productivity, and the oil produced has excellent functional properties (Mozzon et al. 2013). This plant has been cultivated in Asia, Central America, and South America (Rutz et al. 2017). Oil palm plantations consist of three types, namely Dura, Psifera, and Tenera (Umerie et al. 2004). Tenera is a hybrid of Dura and Psifera species that are commonly used in commercial plantations (Cheng

${ }^{*}$ Corresponding author:

Email: dwisetya.sbrc@gmail.com 
et al. 2011). Oval-shaped oil palm fruit is attached to bunches weighing around $10-40 \mathrm{~kg}$, depending on the size and age of plants weighing about 6-20 g per fruit (Ali et al. 2014).

Palm fruit produces two types of oil, namely palm oil and palm kernel oil. Palm oil is produced from the pressing and extraction process of palm fruit mesocarp parts while palm kernel oil is produced from pressing and extracting the palm kernel parts (Tan et al. 2009, Cheng et al. 2011; Ali et al. 2014; Rutz et al. 2017; Albuquerque et al. 2017 2018). Fruit maturity is an essential factor in determining the quantity and quality of oil, especially free fatty acids (FFA) (Saeed et al. 2012, Makky and Soni 2014; Hasibuan, 2016a). Ripe fruit has a higher oil content than raw fruit (Hasibuan, 2016a). Also, the condition of palm oil fruits affects the quality of the oil. Bruises tend to produce oil with higher levels of FFA. High FFA levels also cannot be avoided if it involves a wet process because of the presence of water, which causes hydrolysis of oil to become FFA (Cheng et al. 2011). Palm oil contains triglycerides as the main component and other minor ones, about $1 \%$ (Mohamad et al. 2017). Carotenoids, tocopherols, tocotrienols, sterols, tri-terpenic alcohols, phospholipids, glycolipids, terpenic hydrocarbons, and aliphatics are amongst the minor components found in the palm oil (Umerie et al. 2004; Ayustaningwarno 2012; Silva et al. 2014; Riyadi et al. 2016). Carotene and tocopherol influence the stability and nutrition of palm oil (Umerie et al. 2004).

The word "crude" in palm oil does not mean bad, but it merely indicates raw material that is going to be refined for industrial usages in the production of food products, such as cooking oil, shortening, margarine, cocoa butter substitute (CBS), cocoa butter equivalent (CBE) (Elisabeth 2009; Ayustaningwarno 2012) and non-food products, such as biolubricants, biodiesel, green energy, soap, foaming agent (Cukalovic et al. 2013, Tres et al. 2013, Mba et al. 2015, Albuquerque et al. 2018, Hambali et al. 2019). Palm oil can also be used in functional and nutritional food because of its high phytonutrient contents, such as carotene, tocopherol, and tocotrienol.

The use of palm oil as a food product material needs to be processed first (refinery) to eliminate undesirable components, such as phospholipids, FFA, oxidation products, odors, and colors (Silva et al. 2014; Hasibuan and Siahaan, 2014; Azmi et al. 2015, Hambali et al. 2019). Palm oil refinery can be done chemically and physically, but what is commonly done in the industry is the physical refinery process. The refinery process aims to prevent high neutral oil (triglyceride) losses. The physical refinery process consists of degumming, bleaching, and deacidification (Silva et al. 2014). Degumming and bleaching aim to eliminate phospholipids (through precipitation), and carotenoids (through adsorption). Deacidification removes FFA through volatilization at high temperatures $\left(>200^{\circ} \mathrm{C}\right)$ and low pressure ( $<5$ mbar). During the deacidification, carotenoids are degraded thermally at 240 ${ }^{\circ} \mathrm{C}$ (Ribeiro et al. 2018) so that the color and most of tocopherol and tocotrienol will be lost (Zahrina et al. 2018).

The refinery process produces a product called refined bleached deodorized palm oil (RBDPO) with FFA levels $<0,1 \%$ (Tan et al. 2009). Refinery products in the industry have characteristics, namely bright yellow color, soft taste, and excellent oxidative stability. This refined palm oil is preferred by the community for food products, such as cooking oil because it is considered a higher quality refined oil compared to reddish-orange oil (Yi et al. 2011). This is also caused by the pattern of consumption of cooking oil in Indone- 
sia who is accustomed to clear to yellow coconut oil (Sinaga, 2019). The refinery process also prevents the oil from being oxidized excessively (Schex et al. 2018). However, the refinery process causes minor compounds, such as carotene, tocopherol, and tocotrienol, to be degraded and lost. Even though these compounds are very beneficial for health, such as carotene as pro-vitamin $A$, and tocopherol and tocotrienol as vitamin $\mathrm{E}$ (Hasibuan, 2012; Hasibuan and Siahaan, 2014). Margarine of the red palm oil is potential for functional food as a source of fat, reddish color, and provitamin $A$ and vitamin E. This paper aims to review the development of information about processing, characteristics, and potential use of red palm oil, especially as margarine products.

\section{PRODUCTION AND CHARACTERIS- TICS OF PALM OIL}

Palm oil processing in several countries in Africa is still carried out conventionally. The basic principles in the production of crude palm oil (CPO) in large industries such as Indonesia and Malaysia is in line with that traditional method but wirth significant improvement to meet the need of palm oil based downstream industries. CPO production is a complex process with many variables that affect product quality such as natural variations in fruit, interactions between chemicals, processes, and process changes (Rashid et al. 2017). High-quality palm oil can be produced by controlling the factors that cause quality changes so that the phytonutrient components (especially carotenoids, tocopherols, and tocotrienols) remain high with low FFA levels. In its processing in Africa, palm oil is packaged in packages of varying sizes, shapes, and colors and labeled with packaging which is then marketed in traditional markets and supermarkets and even exports. Palm oil in several countries such as Africa, Southeast Asia, and South America is used directly in cooking and making food. Palm oil is used as a major and minor component in several processed foods (Mba et al. 2015).

In Africa, oil from palm fruit also known as virgin red palm oil (VRPO). VRPO is extracted directly from the palm fruit through a mechanical process by low temperature heating and without the addition of chemicals in its processing. According to Codex Alimentarius (2001), virgin oil is obtained without changing the properties of the oil, namely through mechanical processes such as expeller, press and heat application, and purification by washing using water, precipitation, filtering, and centrifugation. Virgin oil is not allowed to be given additional food additives to it. In addition, according to BPOM (2019), virgin oil is an oil product that is obtained mechanically (by pressing) without heating or by heating a low heat at a temperature of less than $60{ }^{\circ} \mathrm{C}$ and without the use of chemicals. It is important to note that the composition of fatty acids in VRPO is still intact.

\section{Palm Oil Production}

The stages of the palm oil processing process including receiving fresh fruit bunches (FFB), sterilization, threshing, pulverizing, clarifying, purifying, removing water, and storing (Badmus et al. 2005; Ali et al. 2014). In conventional palm oil processing, FFB is sterilized using saturated steam at a pressure of $40 \mathrm{psi}$ (temperature $140 \circ \mathrm{o}$ ) for $75-90$ minutes (Pootao and Kanjanapongkul 2016). The aim is to deactivate the oil hydrolyzing enzymes and maximize the hydrolysis of carbohydrates in FFB (Loi et al. 2011), soften the fruit, and encourage the release of fruit from its bunches (Ariffin et al. 2014). In ad- 
dition to conventional methods, there are several alternative methods to improve oil quality that can be used as pretreatment for oil palm fruit such as continuous sterilization, dry heating, and microwave heating (Pootao and Kanjanapongkul 2016).

Palm fruit is separated from bunches and then softened in a digester and pressed (Tan et al. 2009). During pressing, palm oil extraction from mesocarp leaves about 5-6 \% oil in the fiber material (Wenten et al. 2019). The main pressing tools commonly used are press screw digester, digester with a separate hydraulic press, and digester with press spindle (Azmi et al. 2015). Palm oil is mechanically extracted from palm fruit mesocarp (Makky and Soni 2013). There are two types of palm oil extraction methods, namely wet and dry extraction. In the wet-extraction mode, water is used to extract oil from palm fruit. Hot water or steam is used to release oil from palm fruit cells and to hydrolyze resins, starches, and coagulate proteins. Hydrolyzed and coagulated products are removed during oil clarification. In dry extraction, hydraulic pressure or centrifugation is used (Wenten et al. 2019). Oil from the press is clarified to remove impurities, fibrous material, and non-greasy solids. Hot water is added during clarification so that solids and those that dissolve in water can be separated due to differences in density (Cheng et al. 2011). The oil is then separated and evaporated to reduce the water content.

\section{Palm Oil Characteristics}

The quality parameters of commercial palm oil are free fatty acid (FFA), oxidation level, color, peroxide value, iodine value, water content, specific gravity, refractive index, and viscosity (Oseni et al. 2002). Based on Codex Alimentarius (2001), virgin oil must have a maximum iron $(\mathrm{Fe})$ content of $5.0 \mathrm{mg} / \mathrm{kg}$, a maxi- mum copper content (Cu) of $0.4 \mathrm{mg} / \mathrm{kg}$, a maximum acid value of $4.0 \mathrm{mg} \mathrm{KOH} / \mathrm{g}$ of oil, and a value peroxide maximum of $15 \mathrm{meq} / \mathrm{kg}$ of oil. CPO is also known as unrefined red palm oil, because of its high carotene content. Mba et al. (2015) have reviewed the physicochemical characteristics of palm oil as presented in Tables 1, 2 , and 3 .

Table 1 presents physicochemical properties of palm oil, such as water content, FFA levels, viscosity, iodine value, peroxide value, and saponification value; fatty acid compositions (palmitic acid, oleic acid, linoleic acid, linolenic acid); glycerides (saturated and unsaturated fatty acids; and minor components (beta-carotene, tocopherol, and tocotrienol).

The composition of the primary fatty acids consists of palmitic, oleic, linoleic, and stearic acids with a ratio of saturated and unsaturated fatty acids close to one, whereas the glyceride composition, is mono-, di-, tri-saturated, and unsaturated (Table 2). According to Mba et al. (2015), palm oil also has minor components in the form of micronutrients, such as carotenoids, tocopherols, and tocotrienols (Table 3). The characteristics of palm oil are relatively different, depending on the variety and quality of the fruit, materials, and equipment as well as the techniques and processing carried out. However, until now there has not been found any reference that states with certainty about the characteristics of virgin red palm oil products.

\section{RPO PRODUCTION}

In contrast to West African countries which are countries of origin for oil palm plants, the people prefer direct consumption of oil from the extraction of palm fruit, namely oil without a refinery process, or unrefined palm oil. Meanwhile, some countries such as Southeast Asia do not 
like the direct consumption of unrefined palm oil because it is red in color and has a less familiar 'palmy' smell because people are more accustomed to consuming cooking oil with a golden yellow color. For this reason, it is necessary to process palm oil from direct extraction (unrefined palm oil) to produce red palm oil (RPO) that still retains phytonutrient compounds. RPO is obtained through pre-processing, deacidification, and deodorization processes (Lee et al. 2018). The production of RPO is generally carried out through a chemical refinery process through the stages of degumming (removing gum and impurities with the addition of phosphoric acid), neutralization, and deacidification (neutralizing FFA by adding $\mathrm{NaOH}$ ), and deodorization (removing 'palmy' odor with moderate heating).

\section{Characteristics of RPO}

RPO has components that resemble $\mathrm{CPO}$, namely the distinctive red color because it contains quite high carotene levels. The quality of the RPO is slightly different from CPO because of an extensively heated during the refinery process (degumming, neutralization, and deodorization), even though the process is conducted at relatively lower temperatures. The characteristics of RPO include physicochemical properties (in the form of water content, free fatty acid content, iodic value, peroxide value, color, and odor), micronutrient composition (carotenoids, tocopherols, and tocotrienols) and fatty acids (palmitic acid, oleic acid, linoleic, and stearic). The characteristics of RPO products that have been produced by several researchers are presented in Tables 5 and 6 .

The further process of RPO is fractionation to separate the liquid fraction (red palm olein) and the solid fraction (red palm stearin). The main target in the
RPO processing process is low FFA content with high carotene content. Table 4 presents some processing conditions and characteristics of RPO products. In contrast with VRPO, RPO 's fatty accids are primarily composed of oleic acid, linoleic acid, linolenic acid, and arachidic acid.

Table 1 Physicochemical properties of palm oil

\begin{tabular}{|c|c|}
\hline Characteristics & Range \\
\hline Apparent density at $50^{\circ} \mathrm{C}(\mathrm{g} / \mathrm{ml})$ & $0.892-0.899$ \\
\hline AOM stability (h) & $53.0-60.0$ \\
\hline Melting point $\left({ }^{\circ} \mathrm{C}\right)$ & $33.0-45.0$ \\
\hline Oxidative stability index at $110^{\circ} \mathrm{C}(\mathrm{h})$ & $16.6-19.0$ \\
\hline Refractive index at $50 \mathrm{oC}$ & $1.449-1.456$ \\
\hline Smoke point $\left({ }^{\circ} \mathrm{C}\right)$ & $230.0-235.0$ \\
\hline Solidification point $\left({ }^{\circ} \mathrm{C}\right)$ & $35.0-42.0$ \\
\hline \multicolumn{2}{|l|}{ Solid fat content (\%) } \\
\hline $10^{\circ} \mathrm{C}$ & $30.0-39.0$ \\
\hline $21,1^{\circ} \mathrm{C}$ & $11.5-17.0$ \\
\hline $26,7^{\circ} \mathrm{C}$ & $8.0-14.0$ \\
\hline $33,3^{\circ} \mathrm{C}$ & $4.0-11.0$ \\
\hline $37,8^{\circ} \mathrm{C}$ & $2.5-9.0$ \\
\hline $40,0^{\circ} \mathrm{C}$ & $2.0-7.0$ \\
\hline Specific gravity at $50 \mathrm{oC}$ & $0.888-0.889$ \\
\hline Viscosity (cP) & $45.0-49.0$ \\
\hline lodine value $(\mathrm{g} / 100 \mathrm{~g})$ & $46.0-56.0$ \\
\hline Free fatty acids (\% FFA as palmitate) & $3.17-5.0$ \\
\hline Peroxide value (meqO2/kg) & $0.1-10$ \\
\hline Anisidine value $(\mathrm{mgKOH} / \mathrm{g})$ & $0.6-4.65$ \\
\hline Saponification value $(\mathrm{mgKOH} / \mathrm{g})$ & $190.0-209.0$ \\
\hline Unsaponifiable matter (\%) & $0.15-0.99$ \\
\hline Total Polar Compounds (\%) & $9.47-19.50$ \\
\hline Total polymer materials (\%) & $0.4-15.0$ \\
\hline Saturated fatty acid, SFA (\%) & $49.9-54.7$ \\
\hline Mono-unsaturated fatty acid, & $37.1-39.2$ \\
\hline \multicolumn{2}{|l|}{ MUFA (\%) } \\
\hline Poly-unsaturated fatty acid, & $8.1-10.5$ \\
\hline \multicolumn{2}{|l|}{ PUFA (\%) } \\
\hline Crystal habit & - \\
\hline
\end{tabular}

Source: Mba et al. 2015

\section{NUTRITION OF RPO FOR HEALTH}

RPO is a derivative product of palm oil that still contains high amounts of carotene (as provitamin A) (Hasibuan and 
ljah 2018a). RPO contains essential micronutrients, including carotenoids (500$700 \mathrm{ppm}$ ), tocopherols and tocotrienols (500-1000 ppm), sterols, lycopene, lutein, unsaturated fatty acids, ubiquinone, phytosterols (326-527 $\mu \mathrm{g} \mathrm{g}$ ), and squalene (200-500 $\mu \mathrm{g} / \mathrm{g}$ ) (Ayustaningwarno 2012; Phoon et al. 2018; Mustapa et al. 2011; Riyadi et al. 2016; Sulihatimarsyila et al. 2019).

Table 2 Characteristics of palm oil fatty acid and glyceride composition

\begin{tabular}{|l|l|}
\hline Characteristics & Range \\
\hline Lipid acids compositions (\%) & \\
\hline Lauric acid (C12:0) & $0.1-1.0$ \\
\hline Myristic acid (C14:0) & $0.9-1.5$ \\
\hline Palmitic acid (C16:0) & $41.8-46.8$ \\
\hline Palmitoleic acid (C16:1) & $0.1-0.3$ \\
\hline Stearic acid (C18:0) & $4.5-5,1$ \\
\hline Oleic acid (C18:1) & $37.3-40.8$ \\
\hline Linoleic acid (C18:2) & $9.1-11.0$ \\
\hline Linoleic acid (C18:3) & $0.4-0.6$ \\
\hline Arachidic acid (C20:0) & $0.2-0.7$ \\
\hline Triglyceride composition (\%) & \\
\hline Trisaturated (SSS) & $0.8-9.0$ \\
\hline Disaturated (SUS) & $38.5-50.3$ \\
\hline Monosaturated (SUU) & $31.8-44.4$ \\
\hline Triunsaturated (UUU) & $4.8-9.8$ \\
\hline Diglyserida (\%) & $3.0-7.6$ \\
\hline
\end{tabular}

Source: Mba et al. 2015

Carotene and tocopherol play a role in increasing oil stability (Riyadi et al. 2016). Besides, carotenoids, tocopherols, and tocotrienols, also have antioxidant effects that act as protection against health problems mediated by reactive oxygen, such as cancer, cardiovascular, neurological, and eye diseases (Sathasivam et al. 2018). The minor component also functions as a natural antioxidant (Neo et al. 2010), which can reduce oxidative stress and function to prevent or delay some chronic degenerative diseases (Ayustaningwarno 2012).
Table 3 Minor components and micronutrients of palm oil

\begin{tabular}{|l|l|}
\hline Component/micronutrient & Range (ppm) \\
\hline Carotenoids & \\
\hline a-carotene & $30.0-35.16$ \\
\hline$\beta$-carotene & $50.0-56.02$ \\
\hline Lycopene & $1.0-1.30$ \\
\hline Carotenoids total & $500-700$ \\
\hline Tocopherol & \\
\hline a-Tocopherol & $129-215$ \\
\hline$\beta$-Tocopherol & $22-37$ \\
\hline g-Tocopherol & $19-32$ \\
\hline $\mathrm{t}$-Tocopherol & $10-16$ \\
\hline Tocopherol total & $500-600$ \\
\hline Tocotrienol & \\
\hline- Tocotrienol & $44-73$ \\
\hline- Tocotrienol & $44-73$ \\
\hline- Tocotrienol & $262-437$ \\
\hline- Tocotrienol & $70-117$ \\
\hline Tocotrienol total & $1000-1200$ \\
\hline Phytosterols & $326-527$ \\
\hline Phospholipids & $5-130$ \\
\hline Squalene & $200-500$ \\
\hline Ubikuinon & $10-80$ \\
\hline Alcohol aliphatic & $100-200$ \\
\hline Alcohol triterpenes & $40-80$ \\
\hline Methyl sterols & $40-80$ \\
\hline Aliphatic hydrocarbons & 50 \\
\hline & \\
\hline & \\
\hline
\end{tabular}

Source : Mba et al. (2015)

Carotenoids are a group of yellow to red pigments. Carotenoids are easily isomerized and oxidized by $\mathrm{O} 2$, light, and high temperatures resulting in loss of color and reduced antioxidant effects, as well as vitamin A activity (Rutz et al. 2017). The main carotenoids contained in RPO are $\alpha$ - and $\beta$-carotene, which act as provitamin A (Lee et al. 2018) and are 15-30 times higher than carrot and tomato retinol values (Choo et al., 2003). Thus, carotene can also be used to overcome the problem of lack of vitamin A, namely as a natural dye and food supplements 
Table 4 RPO processing of CPO

\begin{tabular}{|c|c|c|c|c|c|c|c|}
\hline Parameter & Mas'ud et al. 2008 & Hasrini et al. 2009 & $\begin{array}{l}\text { Ayustaningwam } \\
\text { o et al. } 2012\end{array}$ & $\begin{array}{l}\text { Azmi etal. } \\
2015\end{array}$ & $\begin{array}{l}\text { Riyadi etal. } \\
2016\end{array}$ & $\begin{array}{l}\text { Sumama et al. } \\
2017\end{array}$ & $\begin{array}{l}\text { Hasibuan and } \\
\text { ljah 2018a }\end{array}$ \\
\hline Process & Deacidfication & $\begin{array}{l}\text { Degumming, } \\
\text { neutralization, } \\
\text { fraksionation }\end{array}$ & $\begin{array}{l}\text { Degumming, } \\
\text { deacidification, } \\
\text { deodonization }\end{array}$ & $\begin{array}{l}\text { Deacidificat } \\
\text { ion }\end{array}$ & Deodorization & $\begin{array}{l}\text { Wet degumming, } \\
\text { Neutralization, } \\
\text { fraksionation }\end{array}$ & $\begin{array}{l}\text { Degumming, } \\
\text { neutralization, } \\
\text { deodonization }\end{array}$ \\
\hline $\begin{array}{l}\text { Process } \\
\text { condition }\end{array}$ & $\begin{array}{l}\text { Temperature } 59 \circ \mathrm{C}, 25 \\
\text { minutes, } \mathrm{NaOH} 11.1 \% \\
(16 \circ \mathrm{Be})\end{array}$ & $\begin{array}{l}\text { Degumming: } \\
\mathrm{H}_{3} \mathrm{PO}_{4} 85 \% / \mathrm{L} \\
\mathrm{CPO}, 80{ }^{\circ} \mathrm{C} \\
\text { Neutralization: } \\
\mathrm{NaOH} \text { (according } \\
\text { to } \mathrm{FFA} \text { content in } \\
\mathrm{CPO} \text { ), } 59{ }^{\circ} \mathrm{C}\end{array}$ & $\begin{array}{l}\text { Degumming: } 80 \\
{ }^{\circ} \mathrm{C} \text {, fosfic acid } \\
85 \%(0,15 \% \text { of } \\
\mathrm{CPO} \text { veight) } \\
\text { Deacidification: } \\
\mathrm{NaOH} 16 \text { ' } \mathrm{Be} \\
17.5 \% 61{ }^{\circ} \mathrm{C} \text {, } \\
26 \text { menit } \\
\text { Deodonization: } \\
60^{\circ} \mathrm{C} \text { (vacum), } \\
1 \text { hour. }\end{array}$ & $\begin{array}{l}\text { Membrane } \\
\text { fiber } \\
\text { technology } \\
\text { PVDF } \\
\text { crosslinking } \\
\text { PVA } 100 \\
\text { ppm, } 3 \\
\text { hours }\end{array}$ & $\begin{array}{l}\text { Temperature } \\
140^{\circ} \mathrm{C}, 1 \\
\text { hour }\end{array}$ & $\begin{array}{l}\text { Wet degumming. } \\
80{ }^{\circ} \mathrm{C} \text {, fosfic acid } \\
85 \%(0.09 \% \text { of } \\
\mathrm{CPO} \text { weight, vivi) } \\
\text { Neutralization: } 60 \\
{ }^{\circ} \mathrm{C}, \mathrm{NaOH} 0.1 \%\end{array}$ & $\begin{array}{l}\text { Degumming: } \\
\text { fosfic acid } 0,05 \% \\
\text { of } \mathrm{CPO} \text { veight, } \\
60-70{ }^{\circ} \mathrm{C}, 15 \\
\text { minutes } \\
\text { Neutralization: } \\
\mathrm{NaOH} 14 \% \\
\text { according to } \mathrm{FFA} \\
\text { content in } \mathrm{CPO} \text {, } \\
50^{\circ} \mathrm{C}, 30 \text { minutes } \\
\text { Deodonization: } \\
100{ }^{\circ} \mathrm{C}, 60 \\
\text { minutes }\end{array}$ \\
\hline Yield & $95 \%$ & - & $90.16 \%$ & - & - & $\begin{array}{l}\text { Stearin } 35 \% \text { and } \\
\text { olein } 65 \%\end{array}$ & - \\
\hline $\begin{array}{l}\text { RPO } \\
\text { characterist } \\
\text { ics }\end{array}$ & $\begin{array}{l}\text { FFA content } 0.6 \% \text { total } \\
\text { carotenoid } 390 \text { ppm, } \\
\text { vater content } 0.07 \% \\
\text { peroxide value } 59 \\
\text { meq } / 1000 \mathrm{~g} \text {, iod ine value } \\
46 \text {, saponification velue } \\
194 \text {, colour } L=49.77 \mathrm{a}= \\
+30.32 ; \mathrm{b}=+36.95 ; \\
\text { Yellow }(\mathrm{Y})=10 \text {, red (R) } \\
=20\end{array}$ & $\begin{array}{l}\text { Water content } \\
0.035 \% \text { FFA } \\
0.64 \% \text { peroxide } \\
\text { value } 2.32 \\
\text { mg/100g oil, } \\
\text { iodine value } 52.49 \\
\text { (mg/g), total } \\
\text { carotenoid } 511.31 \\
\text { ppm }\end{array}$ & $\begin{array}{l}\text { Reduce of FFA } \\
96.35 \% \text { and } \\
\text { recovery } \\
\text { carotene } 87.30 \\
\%\end{array}$ & $\begin{array}{l}\text { Reduce of } \\
\text { phosphorus } \\
\text { content and } \\
\text { colour. }\end{array}$ & $\begin{array}{l}\text { Carotene } \\
\text { retention } 70 \\
\% \text { reduction } \\
\text { of peroxide } \\
\text { value and } \\
\text { 'palmy' odor. }\end{array}$ & $\begin{array}{l}\text { Olein fraction with } \\
\text { water content } 0.08 \\
\% \mathrm{FFA} 0.17 \% \\
\text { iodine value } 50.79 \\
\mathrm{~g} \text { b/ } 100 \mathrm{~g} \text {, and } \\
\text { peroxide value } \\
50.79 \mathrm{mg} \text { O/ } 100 \mathrm{~g}\end{array}$ & $\begin{array}{l}\text { FFA content } 0.71 \\
\% \text { water content } \\
0.04 \% \text { total } \\
\text { carotene } 263 \\
\text { ppm, colour } \\
10.2 / 10,2 \\
\text { (red/yellow, at } 1 \\
\text { inch cell), } \\
\text { peroxide value } \\
9.45 \text { meq } / \mathrm{kg}\end{array}$ \\
\hline
\end{tabular}

that are very beneficial for improving health (Rice and Burns, 2010; Manorama, 2014). Red palm olein (RPOI) has been used in various forms of food products as a source of carotene (pro-vitamin A) for children who are deficient in vitamin $A$ (El-Hadad et al. 2011).

Vitamin $E$ plays a role in the body's protection against diseases that are mediated by free radicals and for the prevention of heart attacks (Al-Saqer et al. 2004). Vitamin $\mathrm{E}$ is present in eight isomeric forms, namely four tocopherols and four tocotrienols. Tocotrienol is much better as an antioxidant (Musa et al. 2017) than tocopherol. Palm tocotrienol has blood cholesterol-lowering properties and inhibits cancer cell growth (El-Hadad et al. 2011). However further investigation is needed to asses the impact of increasing the pro- portion of linoleic acid (Omega 6) in RPO do to intentional reduction of saturated for human health.

\section{RPO STABILITY}

The stability of RPO, especially carotene, is relatively low during storage. At room temperature, RPO can last for up to 8 months with a reduction in carotene from $500 \mathrm{ppm}$ to $370.1 \mathrm{ppm}$ (Albuquerque et al. 2018). Increasing the stability of RPO can be done through encapsulation. The encapsulation of RPO in polymers or microcapsules can protect the nutritional benefits as well as increase oil stability (Sathasivam et al. 2018). The encapsulation of RPO without heat makes it possible to increase stability and protect its active components from damage 
Table 5 Physicochemical characteristics of RPO

\begin{tabular}{|c|c|c|c|c|c|c|c|c|}
\hline Component & $\begin{array}{c}\text { Hasibuan } \\
\text { and ljah } \\
2018 a\end{array}$ & $\begin{array}{l}\text { Hasibuan } \\
\text { and Meilano } \\
2018 b\end{array}$ & $\begin{array}{l}\text { Sumama et } \\
\text { al. } 2017\end{array}$ & $\begin{array}{l}\text { Riyadi et al. } \\
2016\end{array}$ & $\begin{array}{l}\text { And arwulan } \\
\text { etal. } 2014\end{array}$ & Azlan et al. 2010 & $\begin{array}{l}\text { Hasrini et al. } \\
2009\end{array}$ & $\begin{array}{l}\text { Oseni } \\
\text { et al. } \\
2002\end{array}$ \\
\hline Water content $(\%)$ & 0.71 & $024 \pm 0.07$ & $0.08-0.14$ & 0 & 0.06 & - & 0.035 & 1.2 \\
\hline FFA content $(\%)$ & - & $0.22 \pm 0.03$ & $0.17-0.22$ & $0.490 \pm 0.15$ & 0.15 & - & 0.64 & 3.7 \\
\hline $\begin{array}{l}\text { lodine value } \\
(\mathrm{g} \mid / 2100 \mathrm{~g})\end{array}$ & - & - & $37.48-52.94$ & - & 57.5 & $60.27 \pm 0.89$ & $\begin{array}{l}52.49 \\
(\mathrm{mg} / \mathrm{g})\end{array}$ & 52 \\
\hline $\begin{array}{l}\text { Saponification velue } \\
(\mathrm{mg} \mathrm{KOH} / \mathrm{g})\end{array}$ & - & - & - & - & - & $200,05 \pm 0,42$ & - & 197 \\
\hline $\begin{array}{l}\text { Peroxide value } \\
\text { (meg/kg) }\end{array}$ & - & 0 & $\begin{array}{c}0.03-0.04 \\
(\mathrm{mg} \mathrm{O} / 100 \mathrm{~g})\end{array}$ & $0.12 \pm 0.03$ & 4.2 & $7.98 \pm 0.01$ & $\begin{array}{l}2.32 \\
\text { (mg/100 g } \\
\text { oil) }\end{array}$ & 0.5 \\
\hline $\begin{array}{l}\text { Acid velue } \\
(\mathrm{mg} \mathrm{KOH/g})\end{array}$ & - & - & - & - & - & $0.84 \pm 0.00$ & - & - \\
\hline Colour, Lovibond & - & - & - & $\begin{array}{c}30 \mathrm{Y} \pm 9.6 \mathrm{R} \\
\left(5 \mathrm{y}^{2}\right)\end{array}$ & $\begin{array}{l}11.8 R \\
\left(3 / 8^{*}\right)\end{array}$ & $\begin{array}{l}\text { L: } 85.77 \pm 0.01 \\
\text { a: }-7.43 \pm 0.01 \\
\text { b: } 0.84 \pm 0.00\end{array}$ & - & Red \\
\hline Odor, intensity & - & - & - & 3.3 & - & - & - & - \\
\hline $\operatorname{Density}(g / L)$ & - & - & - & - & - & - & - & 0.9007 \\
\hline Carotenoid (ppm) & 263 & $519 \pm 4.24$ & - & $375.33 \pm 22.87$ & $\begin{array}{c}290.7 \\
\text { (B-carotene) }\end{array}$ & - & 511.31 & - \\
\hline 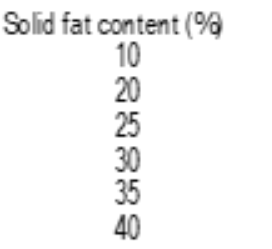 & $\begin{array}{c}52,1 \\
27,1 \\
20,1 \\
10,0 \\
5,1 \\
1,8\end{array}$ & $\begin{array}{l}- \\
- \\
- \\
- \\
-\end{array}$ & $\begin{array}{l}- \\
- \\
- \\
- \\
-\end{array}$ & $\begin{array}{l}- \\
- \\
- \\
- \\
-\end{array}$ & $\begin{array}{l}- \\
- \\
- \\
- \\
-\end{array}$ & $\begin{array}{l}- \\
- \\
- \\
- \\
-\end{array}$ & $\begin{array}{l}- \\
- \\
- \\
- \\
-\end{array}$ & $\begin{array}{l}- \\
- \\
- \\
- \\
-\end{array}$ \\
\hline
\end{tabular}

TL, lightness on scale 0-100 (dari hitam ke putih); a, (+) redness atau (-) greenness; b, yellowness or (-) blueness, C12:0 laurat, C14:0 miristat, C16:0 palmitat, C16:1 palmitoleat, C18:0 stearat, C18:1 oleat, C18:1 elaidat, C18:1 cis-Vaccenat, C18:2 linoleat, C18:3 linolenat, C20:0 arachidat, C20:1 cis-11-eikosanoat, C22:0 behenat, C24:1 lignoserat

during storage and processing (Lee et al. 2018). Yuliasari et al. (2016) encapsulated RPO using a capsule in the form of a mixture of maltodextrin and xanthan gum resulting in encapsulation with $\beta$-carotene content of $3.13226 \mathrm{mg} / \mathrm{kg}, \beta$-carotene retention $72.65 \%$, encapsulation efficiency $72.05 \%$, no oil $1.03 \%$ encapsulated and $92.40 \%$ oil retention.

\section{USE OF RPO IN FOOD PRODUCTS}

RPO has been widely used for food products, including low-temperature cooking oil, margarine, shortening, pow- dered fats, spreads, emulsions in the form of drinks and encapsulation, and other products such as bread, sponge, and biscuits as well as chili. Also, RPO can be used as sachet oil for instant noodles, and salad dressing (Ayustaningwarno 2012, Azlan et al. 2010). A mixture of red palm oil and its fractions or with other vegetable oils is used in various food products such as margarine to soup mixes and infant formulas (Mba et al. 2015). RPO contains beneficial phytonutrients, especially carotene. The carotene is produced without a fortification; therefore, it becomes more cost-effective for the food industry. While 
Table 6 Composition of RPO fatty acids

\begin{tabular}{|c|c|c|c|c|c|c|}
\hline Fatty acid (\%) & $\begin{array}{l}\text { Hasibuan and } \\
\text { ljah 2018a }\end{array}$ & $\begin{array}{l}\text { Ribeiro et } \\
\text { al. } 2018\end{array}$ & Mba et al. 2017 & Azlan et al. 2010 & $\begin{array}{l}\text { Bahurmiz et al. } \\
2007\end{array}$ & $\mathrm{Ng}$ et al. 2003 \\
\hline $\mathrm{C} 12: 0$ & 02 & & & & 0.2 & 0.2 \\
\hline C14:0 & 0.9 & 1.83 & $0.51 \pm 0.04$ & $9.19 \pm 0.03$ & 1.4 & 1.1 \\
\hline C15:0 & & & & $0.91 \pm 0.00$ & & 42.8 \\
\hline $\mathrm{C16:0}$ & 44.7 & 35.43 & $44.32 \pm 0.21$ & $33.84 \pm 0.00$ & 35.8 & - \\
\hline C17:0 & - & - & - & - & - & - \\
\hline $\begin{array}{l}\text { C18:0 } \\
\text { C20:0 }\end{array}$ & $\begin{array}{l}3.9 \\
03\end{array}$ & $\begin{array}{l}11.62 \\
0.37\end{array}$ & $\begin{array}{l}2.85 \pm 0.16 \\
0.09 \pm 0.03\end{array}$ & $3.68 \pm 0.00$ & 3.9 & $\begin{array}{l}3.8 \\
0.3\end{array}$ \\
\hline C21:0 & 0.1 & - & - & $032+000$ & - & - \\
\hline$\frac{\mathrm{C} 24: 0}{\text { Total saturated fatty acid (SFA) }}$ & 50.1 & 49.25 & $47.77 \pm 0.44$ & $\frac{0.32 \pm 0.01}{47.94 \pm 0.02}$ & 41.2 & 48.2 \\
\hline $\mathrm{C} 15: 1$ & & & & & & \\
\hline C16:1 & 0.1 & 0.80 & $0.12 \pm 0.03$ & $0.17 \pm 0.00$ & $1.8(n 7)$ & 0.1 \\
\hline $\begin{array}{l}\text { C17:1 } \\
\text { C18:1 }\end{array}$ & 404 & 3770 & 4) $16+019$ & $3965+0,03$ & 326 & 411 \\
\hline & & $\begin{array}{l}1.10 \\
0.87 \\
0.69\end{array}$ & & & & \\
\hline C19:1 & - & - & - & \multirow{4}{*}{$\begin{array}{c}0.18 \pm 0.02(n 9) \\
- \\
0.14 \pm 0.01\end{array}$} & \multirow{4}{*}{$\begin{array}{l}0 . \overline{(n 9)} \\
0.7(n 11)\end{array}$} & \multirow{4}{*}{-} \\
\hline $\mathrm{C} 20: 1$ & - & - & - & & & \\
\hline C22:1 & - & - & - & & & \\
\hline C24:1 & & & - & & & \\
\hline $\begin{array}{l}\text { Total monounsaturated fatty } \\
\text { acid (MUFA) }\end{array}$ & 40.5 & 40.06 & $48.28 \pm 0.22$ & $40.14 \pm 0.01$ & 35.7 & 41.2 \\
\hline $\mathrm{C} 18: 2$ & 9.3 & 10.10 & $7.76 \pm 0.11$ & \multirow{2}{*}{$\begin{array}{c}0.73 \pm 0.01 \\
10.92 \pm 0.00 \text { (n6) } \\
0.27 \pm 0.02\end{array}$} & $132(n 6)$ & $10.3(n 6)$ \\
\hline C18:3 & 02 & 0.61 & $0.34 \pm 0.07$ & & \multirow{2}{*}{$\begin{array}{c}-(n 6) \\
0.8(n 3) \\
0.3(n 3)\end{array}$} & $0.3(n 3)$ \\
\hline C18:4 & - & - & - & - & & $-(n 3)$ \\
\hline $\mathrm{C} 20: 3$ & - & - & - & - & - & - \\
\hline $\mathrm{C} 20: 4$ & - & - & - & - & \multirow{2}{*}{$\begin{array}{l}0.1(n 6) \\
0.1(n 3) \\
1.6(n 3)\end{array}$} & - \\
\hline $\mathrm{C} 20: 5$ & - & - & $0.88 \pm 0.16$ & - & & $-(n 3)$ \\
\hline $\mathrm{C} 22.5$ & - & - & - & - & \multirow{2}{*}{$\begin{array}{c}-(n 6) \\
0.4(n 3) \\
3.0(n 3)\end{array}$} & - \\
\hline $\mathrm{C} 22: 6$ & - & - & - & - & & - \\
\hline $\begin{array}{c}\text { Total polyunsaturated fatty } \\
\text { acid (PUFA) }\end{array}$ & 9.5 & 10.71 & $8.98 \pm 0.34$ & $11.92 \pm 0.02$ & 19.5 & 10.6 \\
\hline
\end{tabular}

the drawbacks are the reddish color and odor of 'palmy,' which is less liked by consumers, and if used in high-temperature frying can cause the degradation of micronutrient components such as carotene.

\section{Cooking Oil}

Palm cooking oil rich in pro-vitamin A can be obtained by changing the processing conditions to produce oil that still contains pro-vitamin A without having to be fortified with synthetic vitamin A (Hasibuan and Siahaan, 2013). Albuquerque et al. (2018) report that RPO can be used as a cooking oil. Budiyanto et al. (2010) also state that RPO can be used to saute/fry at temperatures less than $150{ }^{\circ} \mathrm{C}$ with short heating. Hasibuan and Meilano (2018b) also used RPO and its olein fraction for stirring media, as a substitute for cooking oil in the cooking of red chili sauce.

Mixing of RPO with coconut oil (80:20) and RPO with a flavor of $0.5 \%$ for frying have been reported by Hasibuan and ljah (2018a). The mixture of RPO has high-quality stability during repeated frying (Hasibuan and ljah, 2018a). Mba et al. (2017) also mixed palm oil with canola oil, which is used as cooking oil. The use of palm oil can be used as a frying media 
with products that do not absorb oil, rich in carotene, and vitamin E. Besides, carotenoids and vitamins contained in palm oil function as natural antioxidants and provide oxidative stability to palm oil during the frying process (Mba et al. 2015).

\section{Shortening}

Hasibuan et al. (2018c) use RPO and unrefined palm oil in making baking shortening as an ingredient for making sweet bread, donuts, and roll cakes. Sweetbread with baking shortening made from RPO and unrefined palm oil has characteristics including water content, fat content, FFA content, peroxide value, and the development of dough resembling commercial products but high carotene content. Julita and Damayanti (2002) used RPO as a substitute for shortening in making cookies with the best composition of rice flour cookies (with 80\% RPO substitution and $\alpha$-carotene content of $29408 \mu \mathrm{g}$ per $100 \mathrm{~g}$ ) and cornstarch cookies (with palm oil substitution red $60 \%$ a-carotene levels $12360.35 \mu \mathrm{g}$ per $100 \mathrm{~g}$ ). Robiyansyah et al. (2017) also used a mixture of RPO and cooking oil in making peanut biscuit products with a normal flavorfull, crispy texture, distinctive nutty flavor, and a yellowish color with beta carotene levels of $347.15 \mathrm{ppm}$.

\section{Margarine}

Andarwulan et al. (2014) used margarine made with the addition of RPO and then used it for pound cake and sweet bread. The pound cake sensory test results show that the texture attribute of the moistness becomes stable, and the sweetbread gives the aroma of fermentation with vigorous intensity and improves the texture (moistness and softness). Hasibuan et al. (2018c) also use RPO and unrefined palm oil in the production of margarine. A mixture of RPO or unre- fined palm oil and RBDPO can be used as an alternative in improving the nutrition of bakery products. Hasibuan and Hardika (2015) made chocolate jam for spread products on white bread) from margarine and chocolate blends made from a mixture of RPO and PKO with organoleptic test results showing that the smear product was acceptable to consumers so that it could be produced as a new innovative product that could fill market opportunities in the bakery industry.

\section{Other Food Products}

RPO has been applied to a variety of food products in addition to palm oil commodity products (cooking oil, shortening, and margarine). Hasrini et al. (2009) make product spreads from RPO with product properties in the form of slip melting point (SMP) and solid-fat content (SFC) having the same characteristics as the properties of commercial spreads while maintaining high levels of carotene. Reputra et al. (2015) made carotene-rich fat powder using a mixture of RPO fraction olein and stearin, as well as fully hydrogenated oil with a product carotene content of 167.71 ppm.

RPO as an emulsion beverage is also very possible to do (Ayustaningwarno 2012). Astuti et al. (2010) have used RPO as a functional ingredient in drinks producing products containing $\beta$-carotene of 180-360 $\mu \mathrm{g} / 250 \mathrm{~g}$. Mursalin et al. (2014) applied RPO to emulsion beverage products. Dwiyanti et al. (2013) have used RPO as an additive in making coconut sugar to improve nutrition. The use of RPO as a supplement to help the work of oral hyperglycemia drugs has also been reviewed by Sinaga et al. (2018). 


\section{POTENTIAL DEVELOPMENT OF RPO AS FOOD PRODUCTS}

RPO has the potential to be developed for a variety of food products in Indonesia. Increasing the preference for food products containing RPO can be done through the nutritional approach of RPO to improve health. Health promotion as a result of the consumption of RPO-based products will cause an increase in public use of products made from RPO. Of the several products described above, one of the products that have the opportunity to be developed from RPO is margarine. This is because margarine products have a reddish color and are semi-solid. In the manufacture of commercial margarine, carotene addition is usually done so that the reddish color. The use of RPO is also a coloring agent of carotene (as pro-vita$\min A$ ) and vitamin $E$ (from tocopherol and tocotrienol). Natural carotenoids are increasingly used in food technology, especially in maintaining consumer safety with regulations that restrict the use of artificial dyes (Davarnejad et al. 2008).

Margarine made from RPO is a product that can provide oxidative stability, texture, and good structure. The use of RPO in the production of margarine is considered beneficial because of its high carotenoid content and gives a natural color to the margarine product produced (Andarwulan et al. 2014). Margarine is also commonly used as a spread product on bread or as a filler in food products (sponge, cake, and sweet bread), so it is more protected from high-temperature heating, which can degrade the phytonutrient components.

\section{CONCLUSION}

Red palm oil (RPO) can be produced from unrefined palm oil or crude palm oil (CPO) using refinery process in- cluding degumming, neutralization, and deodorization at low temperature. The aims of refinery of CPO at low temperatures are reduce the relatively high FFA levels while maintaining phytonutrient levels, through the stages of degumming, neutralization, and deodorization processes. RPO have almost the same characteristics with $\mathrm{CPO}$ in terms of physicochemical properties and minor components, but RPO has lower FFA and carotene levels because it has gone through the refinery stage. RPO has low content of saturated fats as a result of fractionation. RPO can be applied to food products such as cooking oil, shortening, spreads, cooking oil, salad dressings, and margarine. One product that has the potential to be developed based on RPO in Indonesia is margarine because it can act as a source of fat and also provides natural coloring (reddish) and high nutrition from phytonutrient components, especially its carotene content. Margarine is generally used as a spread product on white bread or as a filler in food products (such as sponge cake, cake, and sweet bread). This can help maintain product phytonutrient levels such as carotene in margarine because it is not used at high temperatures which can degrade these nutrient components.

\section{REFERENCES}

Albuquerque T.G., H.S. Costa, M.A. Silva. 2018. Are chloropropanols and glycidyl fatty acid esters a matter of concern in palm oil?. Trends in Food Science and Technology. Doi: 10.1016/j. tifs.2019.01.005.

Ali F.S., R. Shamsudin, R. Yunus. 2014. The effect of storage time of chopped oil palm fruit bunches on the palm oil quality. Agriculture and Agricultural Science Procedia. 2: 165-172. Doi: 10.1016/j.aaspro.2014.11.024 
Al-Saqer J.M., J.S. Sidhu, S.N. Al-Hooti, H.A. Al-Amiri, A.Al-Othman, L. Al-Haji, N. Ahmed, I.B. Mansour, J. Minal. 2004. Developing functional foods using red palm olein. IV. Tocopherols and tocotrienols. Food Chemistry. 85: 579-583. Doi: 10.1016/j.foodchem.2003.08.003.

Alyas, S.A., A. Abdullah, and N.A. Idris. 2006. Changes of $\beta$-carotene content during heating of red palm olein. Journal of Palm Oil Research. Special Issue-April, 2006. P. 99-102.

Andarwulan N., D.R. Adawiyah, N. Wulandari, P. Hariyadi, R.N. Triana, A.R. Affandi, R.C. Nur, S. Tjahjadi, M.F. Ellen, 2014. Aplikasi margarin minyak sawit merah pada produk pound cake dan roti manis. Prosiding Seminar Hasil-Hasil PPM IPB. 1: 192-206.

Ariffin A.A., H.M. Ghazali, P. Kavousi. 2014. Validation of an HPLC method for the determination of hydroxymethylfurfural in crude palm oil. Food Chemistry. 154: 102-107. Doi: 10.1016/j. foodchem.2013.12.082.

Astuti S.D., N. Andarwulan, P. Hariyadi. 2010. Formulasi dan karakterisasi gel minyak sawit (palm oil gel) kaya karotenoid sebagai ingredient pangan fungsional.

Ayeleso, A.O. O.O. Oguntibeju and N.L. Brooks. 2012. Effects of dietary intake of red palm oil on fatty acid composition and lipid profiles in male Wistar rats. African Journal of Biotechnology. 11(33): 8275-8279.

Ayustaningwarno F. 2012. Proses pengolahan dan aplikasi minyak sawit merah pada industri pangan. Vitasphere. 2: 1-11.

Azlan A., K.N. Prasad, H.E. Khoo. N. Abdul-Aziz, A. Mohamad, A. Ismail, Z. Amom. 2010. Comparison of fatty acids, vitamin $\mathrm{E}$, and physicochemical properties of Canarium odontophyllum Miq. (dabai), olive, and palm oils. Journal of Food Composition and
Analysis. 23: 772-776. Doi: 10.1016/j. jfca.2010.03.026.

Azmi R.A., P.S. Goh, A.F. Ismail, W.J. Lau, B.C. Ng, N.H. Othman, A.M. Noor, M.S.A. Yusoff. 2015. Deacidification of crude palm oil using PVA-crosslinked PVDF membrane. Journal of Food Engineering. 166: 165-173. Doi: 10.1016/j.jfoodeng.2015.06.001.

Badan Pengawas Obat dan Makanan (BPOM). 2019. Peraturan badan pengawas obat dan makanan nomor 34 tahun 2019 tentang kategori pangan.

Badmus G.A., N.A. Adeyemi, O.K. Owolarafe. 2005. Performance evaluation of an oil palm fruit screen. Journal of Food Engineering. 69:173-176. Doi: 10.1016/j.jfoodeng.2004.08.011.

Bahurmiz O.M., and W.K. Ng. 2007. Effect of dietary palm oil source on growth, tissue fatty acid composition, and nutrient digestibility of red hybrid tilapia, Oreochromis sp., raised from stocking to marketable size. Aquaculture. 262: 382-392. Doi: 10.1016/j.aquaculture.2006.11.023.

Budiyanto, D. Silsia, Z. Efendi, R. Janika.2010. Perubahan kandungan $\beta$-karoten, asam lemak bebas dan bilangan peroksida minyak sawit merah selama pemanasan. Agritech. 30(2).

Cheng S.F., M.Nor L., C.H. Chuah. 2011. Microwave pretreatment: a clean and dry method for palm oil production. Industrial Crops and Products. 34: 967-971. Doi: 10.1016/j.indcrop.2011.03.002.

Choo. Y.M., H.L.L. Nang, M.A. Ngan, and Y. Basiron. 2003. Extraction of palm vitamin $E$, phytosterol, and squalene from palm oil. The US. Patent 20050250953 A1.

Codex Alimentarius. 2001. Codex Standard for named vegetable oils. Codex Stan 210-1999. Volume 8. 2001.

Cukalovic A., J.C.M. Monbaliu, Y. Eeckhout, C. Echim, R. Verhe, G. Heynder- 
ickx, C.V. Stevens. 2013. Development, optimization and scale up of biodiesel production from crude palm oil and effective use in developing countries. Biomasss and Bionergy. 56: 62-69. Doi: 10.1016/j.biombioe.2013.04.015.

Davarnejad R., K.M. Kassim, A. Zainal, S.A. Sata. 2008. Supercritical fluid extraction of $\beta$-carotene from crude palm oil using CO2. Journal of Food Engineering. 89: 472-478. Doi: 10.1016/j. jfoodeng.2008.05.032.

Dwiyanti H., H. Riyadi, Rimbawan, E. Damayanthi, A. Sulaeman, E. Handharyani. 2013. Efek pemberian gula kelapa yang diperkaya minyak sawit merah terhadap peningkatan berat badan dan kadar retinol serum tikus defisiensi vitamin A. Penelitian Gizi dan Makanan. 36(1): 73-81.

El-Hadad N.N.M., M.M. Youssef, M.H. Abd El-Aal, H.H. Abou-Gharbia. 2011. The utilization of red palm olein in formulating functional chocolate spread. Food Chemistry. 124: 285-290. Doi: 10.1016/j.foodchem.2010.06.034.

Elisabeth, J.E. 2009. Pengalaman Industri Kelapa Sawit dalam Diversifikasi Produk Olahan Hilir. Prosiding Pertemuan Teknis Kelapa Sawit 2009. Jakarta Convention Centre 28 - 30 Mei 2009. Hal. 95 - 103.

Hambali E, Al Sutanto, M Rivai, A Suryani, 2019. Teknologi pengolahan CPO dan produk turunannya. IPB Press. Bogor. Indonesia.

Hasibuan, H. A. 2012. Kajian Mutu dan Karakteristik Minyak Sawit Indonesia serta Produk Fraksinasinya. Jurnal Standardisasi, 14, 13-21.

Hasibuan, H.A., dan D. Siahaan. 2013. Review standar minyak goring sawit diperkaya karoten terkait fortifikasi vitamin A sebagai revisi SNI 01-37412002. Jurnal Standarisasi. 16(1): 6576.

Hasibuan, H.A., dan D. Siahaan. 2014.
Review Standar Minyak Goreng Sawit Diperkaya Karoten Terkait Fortifikasi Vitamin A Sebagai Revisi SNI 0313741-2002. Jurnal Standardisasi, Majalah IImiah Standardisasi. 16: 65-76.

Hasibuan, H.A. dan A.P. Hardika. 2015. Formulasi margarin dan cokelat tabor berbahan minyak sawit dan minyak inti sawit menjadi produk olesan untuk roti tawar. Warta IHP/ Journal of Agrobased Industry. 32 (2): 45-50.

Hasibuan, H.A. 2016a. Pengaruh Penundaan Waktu Pengolahan Buah Sawit Terhadap Berat, Rendemen Crude Palm Oil (CPO) \& Kernel Serta Mutu CPO. Warta Pusat Penelitian Kelapa Sawit. 20 (1): 27-36.

Hasibuan, H.A., 2016b. Retensi Karoten dan Retinol Palmitat pada Minyak Goreng dan Produk Gorengannya. Jurnal Penelitian Kelapa Sawit. 24(3): 147-159.

Hasibuan H.A. dan ljah. 2018a. Peningkatan kesukaan minyak sawit merah dengan penambahan minyak nabati atau flavor dan stabilitasnya dalam penggorengan berulang. Jurnal Penelitian Kelapa Sawit. 26(1): 1-9.

Hasibuan H.A. dan R. Meilano. 2018b. Penggunaan minyak sawit merah dalam pembuatan sambal cabai merah tumis. Jurnal Teknologi Pertanian. 19(2): 95-106.

Hasibuan H.A., A. Akram, P. Putri, E.C. Mentari, B.T. Rangkuti. 2018c. Pembuatan margarin dan baking shortening berbasis minyak sawit merah dan aplikasinya dalam produk bakery. Agritech. 38(4): 353-363.

Hasrini R.F., Sugiyono, P. Hariyadi, N. Andarwulan. 2009. Interesterifikasi enzimatik dengan lipase pada campuran minyak sawit merah dan minyak kelapa untuk menghasilkan bahan baku spreads kaya $\beta$-karoten. Warta IHP/ Journal of Agro-Based Industry. 26(2): 25-36. 
Julita V dan E. Damayanti. 2002. Substitusi minyak sawit merah dan tepung tempe sebagai sumber vitamin A pada produk cookies. Sagu. 1(1): 27-35.

Lee W.J., C.P. Tan, R. Sulaiman, R.L. Smith Jr., G.H. Chong. 2018. Microencapsulation of red palm oil as an oil-in-water emulsion with supercritical carbon dioxide solution-enhanced dispersion. Journal of Food Engineering. 222: 100-109. Doi: 10.1016/j.jfoodeng.2017.11.011.

Loi C.C., H.C. Boo, A.S. Mohammed, A.A. Ariffin. 2011. A high-performance liquid chromatography method for the determination of furfural in crude palm oil. Food Chemistry. 128: 223-226. Doi: 10.1016/j.foodchem.2010.12.108.

Makky M. and P. Soni. 2013. Development of an automatic grading machine for oil palm fresh fruit bunches (FFBs) based on machine vision. Computers and electronics in Agriculture. 93: 129-139. Doi: 10.1016/j.compag.2013.02.008.

Makky M. and P. Soni. 2014. In situ quality assessment of intact oil palm fresh fruit bunches using rapid portable non-contact and non-destructive approach. Journal of Food Engineering. 120: 248-259. Doi: 10.1016/j.jfoodeng.2013.08.011.

Manorama, K. 2014. The potential use of red palm oil in combating vitamin $A$ deficiency in India. Indian Journal of Community Health. 26(1): 45-53.

Mas'ud F, T.R. Muchtadi, P. Hariyadi, T. Haryati. 2008. Optimasi proses deasidifikasi minyak sawit untuk meminimalkan kerusakan karotenoid dalam pemurnian minyak sawit (Elaeis guineensis, Jacq). Forum Pascasarjana. 31(1): 25-36.

Mba O.I., M.J. Dumont, M. Ngadi. 2015. Palm oil: Processing, characterization, and utilization in the food industry- a review. Food BioScience. 10: 26-41. Doi : 10.1016/j.fbio.2015.01.003.
Mba O.I., M.J. Dumont, M. Ngadi. 2017. Thermostability and degradation kinetics of tocochromanols and carotenoids in palm oil, canola oil, and their blends during deep-fat frying. LWT-Food Science and Technology. 82: 131-138. Doi: 10.1016/j.Iwt.2017.04.027.

Mba OI, M. Dumoni, M. Ngadi. 2018. Characterization of tocopherols, tocotrienols and total carotenoids in deepfat fried French fries. Journal of Food Composition and Analysis. 69: 78-86.

Mohamad W.A.F.W., R. Buckow, M. Augustin, D. McNaughton. 2017. In situ quantification of $\beta$-carotene partitioning in oil-in-water emulsions by confocal Raman microscopy. Food Chemistry. 233: 197-203. Doi: 10.1016/j.foodchem.2017.04.086.

Mozzon M., D. Pacetti, P. Lucci, M. Balzano, N.G. Frega. 2013. Crude palm oil from interspecific hybrid Elaeis oleifera $x$ Elaeis guineensis : fatty acid region distribution and molecular species of glycerides. Food Chemistry. 141: 245-252. Doi: 10.1016/j.foodchem.2013.03.016.

Mursalin, Surhaini, A. Yulia. 2014. Stabilitas termal minuman emulsi dari pekatan karoten minyak sawit merah selama penyimpanan. Konversi. 3(1).

Musa I., H. Khaza'ai, M.S.A. Mutalib, F. Yusuf. 2017. Effects of oil palm tocotrienol rich fraction on the viability and morphology of astrocytes injured with glutamate. Food bioscience. 168-177. Doi: /10.1016/j.fbio.2017.10.005.

Mustapa A.N., Z.A. Manan, C.Y.M. Azizi, W.B. Setianto, A.K.M. Omar. 2011. Extraction of $\beta$-carotenes from palm oil mesocarp using sub-critical R134a. Food Chemistry. 125: 262-267. Doi: 10.1016/j.foodchem.2010.08.042.

Neo Y.P., A. Arifin, C.P. Tan, Y.A. Tan. 2010. Phenolic acid analysis and antioxidant activity assessment of oil palm (E. guineensis) fruit extracts. 
Food Chemistry. 122: 353-359. Doi: 10.1016/j.foodchem.2010.02.046.

Ng W.K., P.K. Lim, P.L. Boey. 2003. Dietary lipid and palm oil source affect growth, fatty acid composition, and muscle a-tocopherol concentration of African catfish, Clarias gariepinus. Aquaculture. 215: 229-243.

Oseni K., Owolarafe, Michael O., Faborode. Obafemi O. Ajibola. 2002. Comparative evaluation of the digester-screw press and a hand-operated hydraulic press for palm fruit processing. Journal of Food Engineering. 52: 249-255.

Phoon K.Y., H.S. Ng, R. Zakaria, H.S. Yim, M.N. Mokhtar. 2018. Enrichment of minor components from crude palm oil and palm-pressed mesocarp fiber oil via sequential adsorption-desorption strategy. Industrial Crops and Products. 113: 187-195. Doi: 10.1016/j. indcrop.2018.01.039.

Pootao S. and K. Kanjanapongkul. 2016. Effects of ohmic pretreatment on crude palm oil yield and key qualities. Journal of Food Engineering. 190: 94-100. Doi: 10.1016/j.jfoodeng.2016.06.021.

Rashid N.A., N.A.M. Rosely, M.A.M. Noor, A. Shamsuddin, M.K.A. Hamid, K.A. Ibrahim. 2017. Forecasting of refined palm oil quality using principal component regression. Energy Procedia. 142: 2977-2982. Doi: 10.1016/j.egypro.2017.12.364.

Reputra J., P. Hariyadi, N. Andarwulan. 2015. Penggunaan minyak sawit merah untuk pembuatan lemak bubuk kaya $\beta$-karoten melalui proses pendinginan semprot. Agritech. 35(4).

Ribeiro J.A.A., E.S. Almeida, B.A.D. Neto, P.V. Abdelnur, S. Monteiro. 2018. Identification of carotenoid isomers in crude and bleached palm oils by mass spectrometry. LWT-Food Science and Technology. 89: 631-637. Doi: 10.1016/j. Iwt.2017.11.039.
Rice, A.L. and Burns, J.B. 2010. Moving from efficacy to effectiveness: red palm oil's role in preventing vitamin A deficiency. Journal of the American College of Nutrition 29: 302S-313S.

Riyadi A.H., T.R. Muchtadi, N. Andarwulan, T. Haryati. 2016. Pilot plant study of red palm oil deodorization using moderate temperature. Agriculture and Agricultural Science Procedia. 9: 209-216. Doi: 10.1016/j.aaspro.2016.02.129.

Robiyansyah, A.S. Zuidar, S. Hidayati. 2017. Pemanfaatan minyak sawit merah dalam pembuatan biscuit kacang kaya beta karoten. Jurnal Teknologi Industri dan Hasil Pertanian. 22(1).

Rutz J.K., C.D. Borges, R.C. Zambiazi, M.M.C. Cardozo, L.S. Kuck, C.P.Z. Norena. 2017. Microencapsulation of palm oil by complex coacervation for application in food systems. Food Chemistry. 220: 59-66. Doi: 10.1016/j. foodchem.2016.09.194.

Saeed O.M.B., S. Sankaran, A.R.M. Shariff, H.Z.M. Shafri, R. Ehsani, M.S. Alfatni, M.H.M. Hazir. 2012. Classification of oil palm fresh fruit bunches based on their maturity using a portable fourband sensor system. Computers and electronics in agriculture. 82: 55-60. Doi: 10.1016/j.compag.2011.12.010.

Sathasivam T., S. Muniyandy, L.H. Chuah, P. Janarthanan. 2018. Encapsulation of red palm oil in carboxymethyl sago cellulose beads by emulsification and vibration technology: physicochemical characterization and in vitro digestion. Journal of Food Engineering. 231: 10-21. Doi: 10.1016/j. jfoodeng.2018.03.008.

Schex R., V.M. Lieb, V.M.Jimenez, P. Esquivel. R.M. Schweigert, R. Carle, C.B. Steingass. 2018. HPLC-DAD-APCI/ ESI-MSn analysis of carotenoids and a-tocopherol in Costa Rican Acrocomia aculaeata fruits of varying matu- 
rity stages. Food Research International. 105: 645-653. Doi: 10.1016/j. foodres.2017.11.041.

Silva S.M., K.A. Sampaio, R. Ceriani, R. Verhe, C. Stevens, W.De Greyt. A.J.A. Meirelles. 2014. Effect of type of bleaching earth on the final color of refined palm oil. LWT-Food Science and Technology. 59: 1258-1264. Doi: 10.1016/j.Iwt.2014.05.028.

Sinaga A.G.S., D. Siahaan, K.R. Sinaga. 2018. Potensi minyak sawit merah dan karotenoid sebagai suplemen antioksidan dalam pengujian toleransi glukosa pada tikus putih (Preliminary Study). Seminar IImiah Nasional USU-64: 251256.

Sulihatimarsyila A.W.N., H.L.N. Lau, K.M. Nabilah. I.N. Azreena. 2019. The refining process for the production of refined palm-pressed fiber oil. Industrial Crops and Products. 129: 488-494. Doi: 10.1016/j.indcrop.2018.12.034.

Sumarna D., L.S. Wake, H. SUprapto. 2017. Studi karakteristik minyak sawit merah dari pengolahan konvensional CPO (Crude palm oil). Jurnal Teknologi Pertanian Universitas Mulawarman. 12(2): 35-38.

Tan C.H., H.M. Ghazali, A. Kuntom, C.P. Tan, A.A. Arifin. 2009. Extraction and physicochemical properties of low free fatty acid crude palm oil. Food Chemistry. 113: 645-650. Doi: 10.1016/j.foodchem.2008.07.052.

Tres A., C.R. Samblas, G. van der Veer, S.M. van Ruth. 2013. Geographical provenance of palm oil by fatty acid and volatile compound fingerprinting techniques. Food Chemistry. 137: 142-150. Doi: 10.1016/j.foodchem.2012.09.094.

Umerie S.C., A.S. Ogbuagu, J.O. Ogbuagu. 2004. Stabilization of palm oils by using Ficus exasperate leaves in local processing methods. Bioresource Technology. 94: 307-310. Doi: 10.1016/j.biortech.2004.01.014.
Wenten I.G., A.V. Victoria, G. Tanukusuma, K. Khoiruddin. 2019. Simultaneous clarification and dehydration of crude palm oil using superhydrophobic polypropylene membrane. Journal of Food Engineering. 248: 23-27. Doi: 10.1016/j.jfoodeng.2018.12.010.

Yi J., M.L. Andersen, L.H. Skibsted. 2011. Interactions between tocopherols, tocotrienols, and carotenoids during autoxidation of mixed palm olein and fish oil. Food Chemistry. 127: 1792-1797. Doi: 10.1016/j.foodchem.2011.02.061.

Yuliasari S., D. Fardiaz, N. Andarwulan, S. Yuliani. 2016. Karakteristik enkapsulat minyak sawit merah dengan pengayaan $\beta$-karoten. Informatika Pertanian. 25(1): 107-116.

Zahrina I., M. Nasikin, E. Krisanti, K. Mulia. 2018. Deacidification of palm oil using betaine monohydrate-based natural deep eutectic solvents. Food Chemistry. 240: 490-495. Doi: 10.1016/j.foodchem.2017.07.132. 